\title{
Man versus Machine: the importance of manual blood pressure measurement skills amongst registered nurses
}

\author{
John Unsworth , Guy Tucker, Yvonne Hindmarsh \\ Department of Healthcare, Faculty of Health \& Life Sciences, University of Northumbria, Newcastle-upon-Tyne, United Kingdom
}

Received: April 16, 2015

DOI: $10.5430 /$ jha.v4n6p61

\author{
Accepted: August 17, 2015 \\ Online Published: September 6, 2015 \\ URL: http://dx.doi.org/10.5430/jha.v4n6p61
}

\begin{abstract}
Background: The manual recording of blood pressure is widely accepted to be more accurate than the recording of blood pressure using an automated device. Despite this many western healthcare systems have moved almost entirely to the automated recording of this important vital sign using oscillometric devices. Such devices may either fail to record the patient's blood pressure in persistent hypotension or may give inaccurate readings in people with arteriosclerotic or atherosclerotic changes. This paper explores the importance of manual blood pressure recording, the availability of aneroid sphygmomanometers in UK hospitals and the maintenance of the skills of the workforce following initial nurse education.

Methods: Using a survey of nursing students to explore what opportunities they have to practice manual blood pressures in the clinical setting, the paper explores the maintenance of skills following initial nurse education. The paper also describes the results of data collection, using unobtrusive methods, regarding the availability of aneroid sphygmomanometers in general and specialist hospital facilities in Northern England (UK). Data using both methods were collected in the spring of 2014.

Results: The results suggest that despite most hospitals having some aneriod sphygmomanometers available (mean was 1 device for every 6 beds in acute, 1 device for every 3 beds in specialist hospitals and 1 device for every 12 beds in mental health) they were rarely used in clinical practice with only $35 \%$ of students reporting that they had undertaken a manual blood pressure in a clinical setting during the first year of their course. In addition, some hospitals had no aneroid devices and several others had no central record of location and regular calibration of such devices.

Conclusions: The suggested infrequent use of aneroid devices raises a concern that nurses skills in manual blood pressure recording may be subject to decay over time. Given the importance of these skills in patient safety and, in ensuring accurate and effective care, failure to ensure adequate equipment and opportunities for skill maintenance could result in practitioners and hospitals being open to negligence claims
\end{abstract}

Key Words: Patient safety, Clinical skills, Blood pressure

\section{INTRODUCTION}

Across the world it is widely accepted that the manual recording of blood pressure is more accurate in some patients than the use of automated systems. ${ }^{[1]}$ Despite this most western healthcare systems have moved almost entirely to the use of automatic blood pressure machines for the routine recording of non-invasive blood pressure. There are a number of reasons for this, most notably that the routine taking of vital observations is most commonly undertaken by the least experienced staff, students and healthcare assistants. ${ }^{[2,3]}$

Blood pressure measurement is the cornerstone of vital observations and an essential component of the assessment of a patient's cardiovascular and haemodynamic functioning. While blood pressure measurement is used to detect hyper-

*Correspondence: John Unsworth; Email: john.unsworth@northumbria.ac.uk; Address: Room B105, Faculty of Health \& Life Sciences, Northumbria University, Coach Lane Campus, Newcastle-upon-Tyne, NE7 7XA, United Kingdom. 
tension or hypotension it also provides information about cardiac output, fluid balance and fluid loss. The correct estimation of systolic blood pressure forms a key component of identifying patient deterioration and is one of the consistent measures in physiological track and trigger (early warning scoring) systems used in adults and children. ${ }^{[4]}$

Failure to rescue the deteriorating patient is a global concern. ${ }^{[5,6]}$ In response to the problem of timely identification of patient deteriorating, many western healthcare systems have introduced physiological track and trigger systems (Early Warning Scores - EWS). ${ }^{[7]}$ Irrespective of the field of nursing practice all nurses must be able to measure, record and respond to a patient's vital observations. While patients in adult and children's wards are most likely to be "at risk", environments such as psychiatric and learning disability wards/homes also present opportunities for the detection of physical deteriorating because of illness, rapid tranquillisation, prolonged restraint or because of choking. ${ }^{[8]}$

The gold standard for blood pressure measurement is using an arterial line. Such methods are usually reserved for those individuals who are critically ill because of the inherent risks from arterial catheterisation e.g. infection, embolism. Outside of the critical care setting to routine estimation of blood pressure is undertaken using non-invasive methods. There are two principal methods of non-invasive blood pressure measurement in common use:

\subsection{Auscultation method}

Auscultation (listening) to the blood pressure using a sphygmomanometer and a stethoscope this involves the manual estimation of blood pressure using an aneroid sphygmomanometer. Having selected the appropriate size cuff this is positioned on the patient's upper arm snugly around the patient's arm $2.5 \mathrm{~cm}$ above the anti-cubital fossa (the dip in the arm where the brachial artery is found). The starting point is to estimate the systolic pressure which is done by palpating the Brachial Pulse. This is an important step to avoid operator error in measurement which can include misinterpretation of the auscultatory gap. ${ }^{[9,10]}$ To do this the cuff is rapidly inflated to $20 \mathrm{mmHg}$ (millimetres of Mercury) above the point where the Brachial Pulse disappears. Deflate the cuff and note the pressure at which the pulse reappears this is the estimated systolic pressure. At this point the operator re-inflates the cuff to $20 \mathrm{mmHg}$ above the estimated systolic pressure. The Diaphragm of the Stethoscope is then placed over the Brachial Artery which the cuff is deflated at a rate of 2-3 mmHg per second. The Systolic reading, Korotkoff sound phase one, is noted when the first sound is heard and the last sound is then used as the Diastolic reading. This is the top and bottom figures of the recording for example 120 (systolic) and 62 (diastolic).

\subsection{Oscillometric method}

Oscillometric blood pressure estimation: this is an automatic blood pressure machine which estimates pressure by analysing arterial wall movement. The machine consists of a cuff (with a range of sizes) and a pressure transducer/analyser. The machine automatically pumps air into the cuff until no movement of the arterial wall is detected e.g. the artery is occluded. The pressure is then slowly released until the first movement in the arterial wall is detected (the systolic pressure). Once the arterial wall pressure is equalised the machine uses a complex algorithm to estimate the diastolic blood pressure.

While both methods may be subject to operator error with regard to cuff selection (large cuffs underestimating the pressure and smaller cuffs overestimating) there is a growing body of evidence that oscillometric devices can create errors in certain patient groups. ${ }^{[1,11,12]}$ Errors have been most commonly reported in patients with arteriosclerosis, atherosclerosis but other patient groups may also be at risk of incorrect measurement, these include:

- Diabetics (because of associated atherosclerosis)

- Patients with an arrhythmia

- Patients with excessive movement during measurement e.g. tremor

- Patients with prolonged hypotension

Arteriosclerosis involves a thickening of the artery wall and associated arterial stiffness. ${ }^{[13]}$ As a condition arteriosclerosis has a prolonged latent asymptomatic phase and begins to develop early in adolescence becoming increasingly common by 50 years of age ${ }^{[14]}$ this suggests that blood pressure estimation using oscillometric devices may be inaccurate for a number of patients. Such errors may have important consequences for patient management where the patient is very acutely ill.

In patients with persistent hypotension automatic machines will often produce an error message leading clinicians to assume the blood pressure is un-recordable when in fact a reading may be obtainable via auscultation. It is for this reason that the National Patient Safety Agency ${ }^{[15]}$ recommended to all UK National Health Service (NHS) organisations that staff are taught manual blood pressure recording. Similar recommendations exist in the United States. ${ }^{[9]}$ In addition, the National Patient Safety Agency in a study of reported safety incidents ${ }^{[15]}$ identified that a failure to accurately record vital observations (including blood pressure) was associated with 61 of the 64 deaths reported in 2005. 
Despite the limitations of oscillometric devices and the potential for inaccuracy Smith $^{[9]}$ accepts that automated oscillometric devices are useful where a large number of readings are required e.g. taking the blood pressures routinely of a whole ward full of patients or to avoid the need for expensive training of unqualified staff.

Somewhat surprisingly, given the potential patient safety issues, there have been no previous studies exploring the availability of aneroid sphygmomanometer in UK hospitals and no other published work about the availability or practice of manual recording of blood pressures either in the UK or internationally. The lack of published work sits alongside a dearth of guidance or protocols about the recording of blood pressures in those patient groups where oscillometric devices may produce inaccurate readings.

Many UK Universities providing nurse education programmes have always taught students to measure blood pressure using auscultatory methods. However, in the last decade there has been a move towards assessing students undertaking this skill using an Objective Structured Clinical Examination (OSCE) assessment. An OSCE involves rotating students through a number of stations which simulate a clinical reality. At each station the student is expected to perform a skill on a standardised patient, task trainer or mannequin. ${ }^{[16]}$

Tucker, Unsworth and Hindmarsh ${ }^{[17]}$ have identified how the use of OSCE assessments in under-graduate nurse education is now commonplace. Most of these assessments involve multiple skill based stations and the use of integrated holistic care scenarios as the basis for an OSCE assessment are often only performed towards the end of the course. Within the authors own University an integrated OSCE based assessment designed to assess the skills needed to recognize and organise the appropriate rescue of the deteriorating patient was developed. This assessment has been described in detail elsewhere ${ }^{[17]}$ but it is worth highlighting that the assessment requires students to measure the patient's vital signs, interpret the results, perform a structured handoff using the SBAR (Situation, Background, Assessment and Recommendation) format and then make an appropriate record of the intervention and outcome. Students undertaking the OSCE assessment receive specific instruction during lectures, seminars and practical and they are encouraged to practice their skills prior to the assessment. Anecdotal reports from students suggested that they felt that there was limited scope to practice their skills outside of the University setting because the auscultatory method was not widely used in practice and that many clinical areas did not have the equipment to allow for manual blood pressure measurement. With this in

Published by Sciedu Press mind the aim of this study was to identify the availability of equipment and to explore what opportunities students had to practice the measurement of manual blood pressure prior to and after their OSCE assessment. More specifically the study set out to:

(1) Examine the number of oscillometric devices in use per in-patient bed in NHS hospitals in UK

(2) Examine the number of auscultatory devices in use per in-patient bed in NHS hospitals in UK

(3) Examine what opportunities students have to practice manual blood pressure measurement prior to the OSCE assessment and whether they subsequently practice the skill in practice

\section{Methods}

\subsection{Ethics}

Ethical approval for the study was sought from the Faculty Research Ethics Committee and approval granted prior to the commencement of data collection.

\subsection{Survey instrument}

The study utilized both a survey and unobtrusive methods. A short self-completed survey was provided to all students who agreed to participate in the research. The survey consisted of demographic data exploring the participant's age, gender and previous healthcare experience. In addition, the survey explored what opportunities students had to practice their skills both within University and in the clinical setting both prior to and after the OSCE assessment.

Surveys are instruments which usually consist of a series of questions and/or statements which elicit responses from research subjects. ${ }^{[18]}$ Most surveys are quick to complete, relatively inexpensive to administer and can be distributed to relatively large samples. The disadvantages of surveys include issues around response bias and unreliability of the responses. ${ }^{[19]}$

\subsection{Sample}

The self-completed survey was distributed, in the spring of 2014 , to 325 under-graduate nursing students from one University who were at the end of their first year of their studies. They had all had theoretical and practical instruction about vital observation measurement and had had the opportunity to practice these skills in University and in clinical practice prior to undertaking their OSCE examination. All of the students had successfully completed their OSCE during the course.

\subsection{Unobtrusive methods design}

Unobtrusive methods were used to collect data from NHS hospitals in UK. Unobtrusive methods are described as meth- 
ods where "data is gathered by means that do not involve direct elicitation of information from research subjects". ${ }^{20]}$ Kellehear $^{[21]}$ outlines how the strength of unobtrusive methods is that they often consist of documentation of actual rather than self-reported events or behaviour. In addition, the methods are inexpensive to administer and can be replicated longitudinally. On the other hand weaknesses include misrepresentation of requests for information and manipulation of the data by the respondent to prevent an organisation appearing in a bad light.

One form of unobtrusive method is the use of Freedom of Information Act (2000) requests to public organisations. Within the United Kingdom the Freedom of Information Act (2000) requires public bodies to make available on in advance by via publication scheme specific information about their activities. In addition, individuals can request information in writing and organisations are required to comply with requests within 20 working days of the request being received unless they consider that the information is exempt or disclosure would be prejudicial in some way. Freedom of Information as an unobtrusive research method remains immature partially because of inadequate reporting of the method in studies which have used this approach. ${ }^{[2]}$

\subsection{Unobtrusive method sample}

The research team made written request to 64 NHS hospitals in UK, in the spring of 2014, asking for the following data:

(1) The number of in-patient beds provided by each organisation

(2) The number of electronic non-invasive blood pressure monitors (oscillometric) in use within the organisation

(3) The number of manual aneroid sphygmomanometers in use within in-patient areas within the organisation

For the sake of clarity organisations were informed that inpatient beds included day surgery trolleys, paediatric cots and temporary beds within emergency departments which are used for rapid patient assessment. Each organisation was specifically asked to exclude all aneroid sphygmomanometers in use by community services and community nursing teams.

While Freedom of Information requests can place a considerable burden on public bodies the research team believed that the information requested should have been readily available to NHS organisations as they are required to maintain asset registers of equipment which requires regular servicing, calibration and replacement.

\subsection{Data analysis}

Data from the surveys were analysed using Microsoft Excel to produce descriptive statistics (frequency and percentages) for each of the questions. Data from the unobtrusive methods were analysed to compare the frequency of each type of equipment against the total number of beds within the hospital facility.

\section{Results}

\subsection{Unobtrusive methods}

The unobtrusive methods resulted in a good response rate to the requests for information. Requests were sent to a range of NHS Hospitals in UK (this covered the area of the North East, Yorkshire and the Humber and the North West). These NHS areas served a population of just over 15 million people $(15,086,775)$ and covered a geographical area of 38,177 square kilometres (14,740 square miles). ${ }^{\text {[23] }}$ Hospitals in this study were characterised as being acute general providers, specialist mental health/learning disabilities providers or specialised hospitals. Those hospitals identified as specialised included cancer treatment centres, children's hospitals and specialist cardiac and respiratory centres. A total of 44 requests were made to acute hospitals with a response being obtained from 36 hospitals without a reminder ( $81 \%$ response rate). A total of 13 requested were made to specialist mental health and disability hospitals with 13 responses being received giving a $100 \%$ response rate. Finally, 7 requests were sent to specialist hospitals with 6 responses being provided giving an $85 \%$ response rate.

\subsection{Acute hospitals}

The results for acute hospitals showed that oscillometric devices were more common than aneroid machines in all acute hospitals. The mean was 1 oscillometric blood pressure monitor for every three in-patient beds (range 1 device for every bed to 1 device for every 7.5 beds). One acute hospital indicated that they had no aneroid sphygmomanometers and this organisation was therefore totally reliant upon oscillometric machines. In terms of aneroid (manual) devices the mean was 1 sphygmomanometer for every 6 beds (range 1 for every 1.5 beds to 1 for every 32 beds).

A total of $22 \%$ of respondents $(n=8)$ indicated that they had no central record of aneroid sphygmomanometers despite that requirement that these machines were regularly maintained and calibrated at least every 6 months. ${ }^{[24]}$

\subsection{Mental health and learning disability hospitals}

The results for the mental health and disability hospitals showed that while oscillometric devices were still more common than aneroid sphygmomanometers they were less common than in acute environments with one device for every 12 beds (range 1 for every 6 beds to 1 for every 30 beds). None of the hospitals indicated that they were unable to provide 
the information and there was no hospital which had zero aneroid sphygmomanometers available for use. The mean for aneroid devices was 1 for every 17 . However, one hospital had one aneroid sphygmomanometers for every 234 beds, making the availability of equipment particularly scarce.

\subsection{Specialist hospitals}

The results for the specialist hospital showed that oscillometric devices were again more common with an average of one device for every 3 beds (range 1 for every 1.2 beds to 1 device for every 6.5 beds). Of the specialist hospitals only one had no aneroid sphygmomanometers available at all. The average number of aneroid devices was one for every 12 beds with a range of zero devices per bed to 1 device for every 27 beds.

\subsection{Student survey}

A self-completion survey was distributed to 325 undergraduate nurses all of whom had successfully completed their OSCE assessment. A total of 250 surveys were completed representing an $76 \%$ response rate. The results of the survey showed that the majority of respondents were female $(\mathrm{n}=230,92 \%)$ with 20 males $(8 \%)$ making up the remaining respondents. The mean age of the respondents was 24 years (range 18-47 years) and a total of 93 students (37.2\%) had prior experience of healthcare before commencing on the under-graduate nursing course. Students were asked to indicate what opportunities they had had to practice the manual recording of blood pressures both during both formal taught sessions, in the clinical setting and during informal practice. Students were asked to tick all boxes that applied. Table 1 details the responses. The majority of students had practiced on a mannequin $(n=221,88.4 \%)$ prior to their OSCE examination. A smaller number had practiced on other students, on a patient when in the clinical setting and on staff. Mannequins offer students the opportunity to practice their skills in a safe and lower pressure environment than in the clinical setting. The benefit of a mannequin is that lower and high blood pressures can be set thereby offering an opportunity to practice obtaining these measurements. Such opportunities to record abnormal pressures may not always present themselves in the clinical setting. Even when working alone a student can check whether they have obtained the correct recording by reviewing the mannequin's settings once they have obtained the blood pressure manually. ${ }^{[25]}$

Students were asked to indicate if they had measured manual blood pressure since completing their OSCE examination. A total of 120 students $(48 \%)$ had undertaken to record manual blood pressure since their examination. A variety of reasons for recording manual blood pressure were noted but

Published by Sciedu Press most significantly because an ocillometric device was unable to obtain a reason or because of concerns about the accuracy of the reading because of high or low blood pressure. Table 2 shows the results for this questions and of note is the routine use of manual blood pressure recordings in some areas particularly community and children's nursing settings.

Table 1. Opportunity to practice blood pressure prior to the OSCE assessment*

\begin{tabular}{lll}
\hline & n & Percentage \\
\hline At University on other students & 87 & $34.8 \%$ \\
At University on a mannequin & 221 & $88.4 \%$ \\
In the clinical setting on a patient & 90 & $36 \%$ \\
In the clinical setting on other students & 87 & $34.8 \%$ \\
In the clinical setting on staff & 86 & $34.4 \%$ \\
At home on friends and family & 68 & $27.7 \%$ \\
${ }^{*}$ Students could select more than one category (all students indicated at least \\
one category)
\end{tabular}

Table 2. Use of manual (auscultatory) measurement of blood pressure

\begin{tabular}{lll}
\hline Circumstances & $\begin{array}{l}\text { Number of } \\
\text { Students }\end{array}$ & $\begin{array}{l}\text { Percentage of } \\
\text { Respondents }\end{array}$ \\
\hline $\begin{array}{l}\text { Routinely in the clinical setting } \\
\begin{array}{l}\text { Automated blood pressure machine } \\
\text { could not record / inaccurate reading }\end{array}\end{array}$ & 46 & $35 \%$ \\
$\begin{array}{l}\text { For accuracy in certain patient } \\
\text { conditions }\end{array}$ & 6 & $12 \%$ \\
$\begin{array}{l}\text { On friends and family } \\
\text { Note. Not every student provided the circumstances }\end{array}$ & $4 \%$ \\
\hline
\end{tabular}

Note. Not every student provided the circumstances

Students were asked if they had used any of the skills assessed during the OSCE examination e.g. vital observation measurement (which by definition includes blood pressure measurement), calculation of an early warning score, SBAR handover, identification of patient deterioration and record keeping. Table 3 details the results with almost all students using these skills during the first year of their course whilst in the clinical setting.

\section{Discussion}

From the data presented it can be seen that Aneroid sphygmomanometers widely available in most hospitals. However, ocillometric devises still outweigh the ratio of equipment in favour of electronic over manual equipment across all fields of nursing practice. Mental health and Learning disabilities equipment ratios may differ due to the more physically stable nature of their client group compared to the acute setting. While the unobtrusive data clearly indicates that although hospital Trusts state they have aneroid sphygmomanometers available the survey suggests that they are used infrequently in the practice setting. Indeed the majority of students surveyed practiced the skill of manual auscultation 
of blood pressure away from the clinical setting (at home, at university) with only $36 \%(n=46)$ practicing on a patient in a hospital environment. This suggests that the practice of manually recording a patient's blood pressure is performed infrequently in practice raising questions about how both nursing students and registered nurses can maintain their skills in this area. Furthermore, the survey data appears to suggest that social modelling ${ }^{[26]}$ in the workplace is not occurring and therefore students have little opportunity to see manual auscultation of blood pressure being performed and correspondingly little opportunity to use pro social modelling to emulate "best practice".

Table 3. Use of vital observation and associated skills since OSCE assessment

\begin{tabular}{lllll}
\hline Skill/Component & Yes & Percentage & No & Percentage \\
\hline Taking and recording of vital Observations ${ }^{*}$ & 249 & $99.6 \%$ & 1 & $0.4 \%$ \\
Calculation of an Early Warning Score (EWS) & 242 & $96.8 \%$ & 8 & $3.2 \%$ \\
Identification of patient deterioration & 243 & $97.2 \%$ & 7 & $2.8 \%$ \\
Handover for information to other staff & 243 & $97.2 \%$ & 7 & $2.8 \%$ \\
Record keeping related to observations or deterioration & 249 & $99.6 \%$ & 1 & $0.4 \%$ \\
Care delivery to a deteriorating patient & 232 & $92.8 \%$ & 18 & $7.2 \%$ \\
\hline
\end{tabular}

"Vital observations includes - blood pressure, pulse, respiratory rate, temperature and pulse oximetry

Of greater concern was the fact that a small number of hospitals had no aneroid devices $(n=2)$ at all and a number of hospitals had no way of providing information about the number of devices available $(n=8)$. This raises concerns about the location, maintenance and calibration of these devices as the organisations themselves were unable to locate the items on their asset register. Organisations with few aneroid sphygmomanometers could compromise patient care and safety if there was a need to quickly and accurately measure a blood pressure for example in a patient with persistent hypertension. These organisations are also likely to have considerable problems maintaining the skills of their nursing and healthcare workforce because opportunities to practice auscultatory blood pressure measurement using an aneroid sphygmomanometer will be more limited.

Anecdotal reports, from students, suggest that some registered nurses are unable to manually record a patient's blood pressure. Irrespective of whether or not they currently have the skills the question of potential skill decay remains. Skill decay is the partial or full loss of trained or acquired skills and knowledge following periods of non-use. Studies exploring skills decay (fade) in surgeons have suggested that the skills taught during initial education are still evidence six to nine months after the training ${ }^{[27]}$ and that any skills fade can be repaired by structured practice every six months. ${ }^{[28]}$ This suggests that registered nurses may only need to practice the skill of manually recording a blood pressure two or three times a year to maintain their competence in this area. While the maintenance of competency is associated with the frequency of skills use ${ }^{[29]}$ overlearning of the skill can assist with retention. ${ }^{[30]}$ Loss of critical skills and knowledge is of great concern, and is problematic in situations in which student nurses are receiving the initial training but may not have an opportunity to use these skills or knowledge for extended periods of time. The development of the OSCE assessment and the subsequent dissemination of this work to our local hospitals have resulted in changes in practice with hospital managers purchasing more aneroid sphygmomanometers and introducing update training for their own registered nurses.

\subsection{Limitations}

One of the limitations of this study is that it failed to identify and assess whether registered nurses had the skills in the manual recording of blood pressure. Furthermore, there are limitations to this article, in that there is insufficient data to support the reasons why more students are practicing manual auscultation of blood pressure away from the clinical environment. This may be due to lack of understanding of clinical factors which affect the accuracy of such measurements and equipment as observed by Tucker, Unsworth and Hindmarsh. ${ }^{[17]}$

\subsection{Implications}

Hospital managers have a legal responsibility to ensure staff have adequate resources (aneroid machines) to be able to deliver their duty of care. Furthermore, hospital managers and administrators should be aware of the availability of equipment within their own hospital. This is important because of the need to use such aneroid sphygmomanometers should electronic equipment fail or if it is unable to record an accurate blood pressure due to the factors discussed earlier in this article. One way of further ensuring staff competence could be developing a clear protocol for which category of patients should have a manually recorded blood pressure taken at least once during their hospital stay. If this was then recorded on the observation chart it would be possible to audit compliance with the protocol as well as to ensure that more accurate readings were obtained in certain client groups 
e.g. those with Parkinson's disease or advanced arteriosclerosis or atherosclerosis. In addition, mandatory training of staff, such as annual updates using a competency based framework linked to best evidence, ${ }^{[31]}$ could ensure patient safety whilst protecting against negligence claims.

\section{Conclusion}

This study suggests that hospitals need to assess the availability of aneroid devices within their own health care settings as well as establishing the skill levels of staff. Furthermore, clinical protocols or guidelines may be required to differentiate when the manual recording of blood pressures may be more appropriate than using oscillometric devices. Further research work is needed to establish how frequently registered nurses and other health professionals needs to record blood pressures manually to avoid skill decay.

\section{REFERENCES}

[1] van Popele NM, Bos WJ, de Beer NA, et al. Arterial stiffness as underlying mechanism of disagreement between an oscillometric BP monitor and a sphygmomanometer. Hypertension. 2000 Oct; 36(4): 484-8. PMid: 11040223. http://dx.doi.org/10.1161/01. HYP. 36.4 .484

[2] Smith S. An audit of nursing observations on ward patients. Nursing Times. 2008; 104(30): 28-29.

[3] Watson D. The impact of accurate patient assessment on quality of care. Nursing Times. 2006; 102(6): 32-37.

[4] Royal College of Physicians The National Early Warning Score (NEWS): standardising the assessment of acute illness severity in the NHS. RCP: London; 2012.

[5] Clarke SP, Aiken LH. Failure to rescue. American Journal of Nursing. 2003; 103: 42-47. http://dx.doi.org/10.1097/00000446-2 00301000-00020

[6] Jones S, Bottle A, Griffiths P. An assessment of "failure to rescue" derived from routine NHS data as a nursing sensitive patient safety indicator for surgical inpatient care. National Nursing Research Unit, Kings College: London; 2011.

[7] Jansen JO, Cuthbertson BH. Detecting critical illness outside of the ICU: the role of track and trigger systems. Current Opinion in Critical Care. 2010; 16: 184-190. PMid: 20305556. http: //dx.doi.org/10.1097/MCC.0b013e328338844e

[8] NPSA. Rapid Response Report NPSA/2008/RRR010: Resuscitation in Mental Health and Learning Disability settings. National Patient Safety Agency: London; 2008.

[9] Smith L. New AHA Recommendations for Blood Pressure Measurement. Am Fam Physician. 2005; 72(7): 1391-1398.

[10] Karnath B. Sources of error in blood pressure measurement. Hospital Physician. 2002: 33-37.

[11] Robbin KR. Non-invasive blood pressure monitoring. Critical Care Nursing. 2002; 22(2): 123-124.

[12] Chang JJ, Rabinowitz D, Shea S. Sources of variability in blood pressure measurement using the Dinamap PRO 100 automated oscillometric device. American Journal of Epidemiology. 2003; 158: 1218-1226. PMid: 14652308. http://dx.doi.org/10.1093/aje/kwg274

[13] Jules YTL. Definition of Arteriosclerosis. Merk Manual. 2012 [accessed 06/09/2014]. Available from: http://www.merckmanua ls.com/professional/cardiovascular_disorders/arter iosclerosis/definition_of_arteriosclerosis.html

[14] Insull W. The Pathology of Atherosclerosis: Plaque Development and Plaque Responses to Medical Treatment. The American Journal of Medicine. 2009; 122(1 Suppl): S3-S14. PMid: 19110086. http://dx.doi.org/10.1016/j.amjmed.2008.10.013

[15] NPSA Recognising and responding appropriately to early signs of deterioration in hospitalised patients. National Patient Safety Agency: London; 2007.

Published by Sciedu Press
[16] Merriman C, Westcott L. Succeed in OSCEs and Practical Exams: an essential guide for nurses. MacGraw Hill: London; 2010

[17] Tucker G, Unsworth J, Hindmarsh Y. First Do No Harm: teaching and assessing the recognition and rescue of deteriorating patients to nursing students. Journal of Nurse Education and Practice. 2015; 5(6): 31-38. http://dx.doi.org/10.5430/jnep.v5n6p31

[18] Franklin BJ, Osborne HW (Eds.). Research Methods: Issues and Insights. Wadsworth Publishing Company; 1971.

[19] Oppenheim A. Questionnaire Design, interviewing and Attitude Measurement. London, Pinter; 1992.

[20] Lee RM. Unobtrusive Methods in Social Research. Buckingham: Open University Press; 2000.

[21] Kelleher A. The Unobtrusive Researcher: a guide to methods. Allen and Unwin: Sydney; 1993.

[22] Booth A. Innovative Uses of the Freedom of Information Act (2000) for Research. 2009 [accessed 06/09/2014]. Available from: http: //www.rin.ac.uk/our-work/researcher-development-a nd-skills/freedom-information-tool-researchers-0

[23] Office of National Statistics. Annual mid-point population estimates for Clinical Commissioning Groups. ONS: London; 2013.

[24] British Hypertension Society. Frequently Asked Questions blood pressure monitors. 2012 [accessed 06/09/2014]. Available from: http://www.bhsoc.org/frequently-asked-questio $\mathrm{ns} / \# \mathrm{bpm} 5$

[25] Lateef F. Simulation based learning: just like the real thing. Journal of Emergencies, Trauma and Shock. 2010; 3(4): 348-352. PMid: 21063557. http://dx.doi.org/10.4103/0974-2700.70743

[26] Bandura A. Social learning theory. Englewood Cliffs, NJ: PrenticeHall; 1977.

[27] Enk D, Gilead LC, Smolovich IC, et al. Diagnostic performance and retention of acquired skills after a dermatology elective. International Journal of Dermatology. 2003; 42: 812-815. PMid: 14521697. http://dx.doi.org/10.1046/j.1365-4362.2003.02018.x

[28] Castellvi AO, Hollett LA, Hogg DC, et al. Maintaining proficiency after fundamentals of laproscopic surgery training: a 1-year analysis of skills retention for surgery residents. Surgery. 2009; 146(2): 387-393. PMid: 19628100. http://dx.doi.org/10.1016/j.s urg. 2009.05.009

[29] Numminen O, Meretoja R, Ihoaho H, et al. Professional competence of practicing nurses. Journal of Community Nursing. 2013; 22(10): 1411-1423.

[30] Perez RS, Skinner A, Weyhrauch P, et al. Preventing surgical skills decay. Military Medicine. 2013; 178(10): 76-86. PMid: 24084308. http://dx.doi.org/10.7205/MILMED-D-13-00216

[31] National Institute for Health and Clinical Excellence NICE. Hypertension: The clinical management of primary hypertension in adults. Clinical guideline 127. 2011 [accessed 25/08/14]. Available from: http://nice.org.uk/nicemedia/live/13561/5 $6007 / 56007 . p d f$ 